Case Report

\title{
Nasal Septal Perforation in Propylthiouracil-Induced Anti- Neutrophil Cytoplasmic Antibody-Associated Vasculitis
}

\author{
Yusho Ishii (D), Tsuyoshi Shirai (D), Yousuke Hoshi, Yoko Fujita, Yuko Shirota, Hiroshi Fujii, \\ Tomonori Ishii, and Hideo Harigae
}

Department of Hematology and Rheumatology, Tohoku University Graduate School of Medicine, Sendai, Japan

Correspondence should be addressed to Tsuyoshi Shirai; tsuyoshirajp@med.tohoku.ac.jp

Received 25 August 2017; Revised 13 December 2017; Accepted 8 January 2018; Published 22 March 2018

Academic Editor: Jamal Mikdashi

Copyright (c) 2018 Yusho Ishii et al. This is an open access article distributed under the Creative Commons Attribution License, which permits unrestricted use, distribution, and reproduction in any medium, provided the original work is properly cited.

\begin{abstract}
Here, we present the case of a 29-year-old woman with nasal septal perforation and positive myeloperoxidase- (MPO-) antineutrophil cytoplasmic antibody (ANCA). She had been diagnosed with Graves' disease and had been treated with propylthiouracil (PTU) for 14 months. A biopsy of the nasal septum revealed an infiltration of inflammatory cells, with no evidence of malignancy or granulomatous change. Because of the use of PTU, destructive nasal lesion, and positive MPO-ANCA, she was diagnosed with druginduced ANCA-associated vasculitis (AAV) and was treated with prednisolone and methotrexate after the cessation of PTU. Although PTU is known to be the medicine that induces drug-induced AAV, the manifestation of nasal septal perforation in druginduced AAV is poorly identified. This is the rare case of drug-induced AAV which manifested only nasal septal perforation.
\end{abstract}

\section{Introduction}

Anti-neutrophil cytoplasmic antibody- (ANCA-) associated vasculitis (AAV) generally occurs in middle-aged and older adults, and AAV patients manifest heterogeneous symptoms, such as fever, weight loss, myalgia, skin vasculitis, and sinonasal involvement. AAV involves the inflammation of small and medium arteries, which results in neuropathy, interstitial pneumonia, glomerulonephritis, otitis media, and sinusitis [1]. AAV comprises three different diseases: granulomatosis with polyangiitis (GPA), eosinophilic granulomatosis with polyangiitis, and microscopic polyangiitis [2]. In addition, some drugs cause drug-induced AAV [3]. In particular, propylthiouracil (PTU) and thiamazole, which are used to treat Graves' disease (GD), are known to induce ANCA and AAV $[3,4]$. Here, we report the first case of PTU-induced AAV that manifested with only nasal septal perforation.

\section{Case Presentation}

A 29-year-old woman was referred to our hospital for the evaluation of nasal septal perforation with positive MPOANCA. Fifteen months before referral, she had been diagnosed with GD and consequently treated with PTU for 14 months. Two months before referral, she had developed a lowgrade fever and sore throat and visited the otorhinolaryngology department. The otorhinolaryngologist detected nasal septal perforation and performed biopsy that revealed an infiltration of inflammatory cells into the basal layer of the epidermis, with no evidence of malignancy. In addition, she had never taken cocaine. AAV was suspected because MPO-ANCA was positive, and she was subsequently referred and admitted to our hospital. Because she was taking PTU and drug-induced AAV was suspected, PTU treatment was stopped a month before referral. On admission, thyroid gland swelling was detected. Laboratory findings, including those of urinalysis, blood biochemistry, and coagulation testing as well as complete blood count, were within the normal range. MPO-ANCA was positive $(110 \mathrm{U} / \mathrm{mL}, 3.4$ is a reference value in our hospital), and proteinase 3- (PR3-) ANCA was slightly positive $(3.9 \mathrm{U} / \mathrm{mL}, 3.4$ is a reference value in our hospital). Other autoantibodies were negative, except for anti-thyroid-stimulating hormone (TSH) receptor antibody (7.37 IU/L). Free thyroxine and free triiodothyronine levels were elevated, and the TSH level was decreased. Nasal endoscopy revealed nasal septal perforation (Figure 1). Computed tomography revealed the absence of 


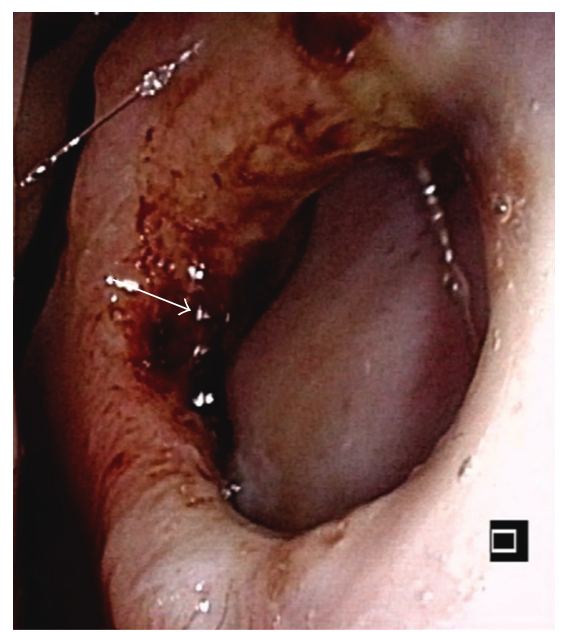

Figure 1: Nasal endoscopic finding. Nasal endoscopy shows nasal septal perforation.

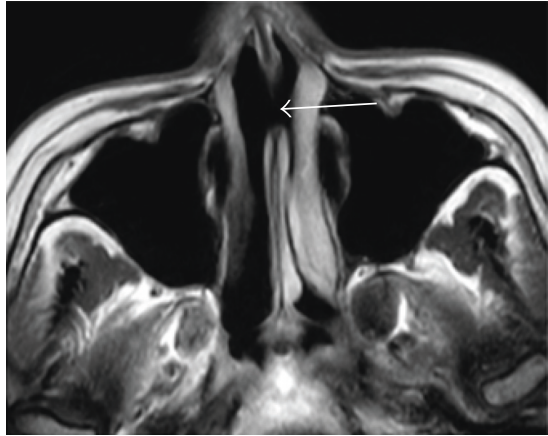

(a)

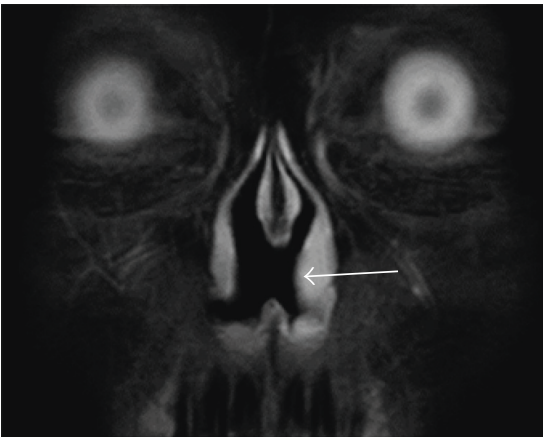

(b)

FIgURE 2: Magnetic resonance imaging (MRI) findings. MRI shows nasal septal perforation in horizontal (a) and coronal (b) sections.

sinusitis and pneumonia. Magnetic resonance imaging (MRI) revealed nasal septal perforation (Figure 2). We again performed a biopsy of the perforated nasal septum for differential diagnosis, which revealed an infiltration of inflammatory cells, including neutrophils and lymphocytes, with no evidence of malignancy or granulomatous change. Together with a destructive nasal lesion and positive MPO-ANCA, she was diagnosed with AAV, particularly PTU-induced AAV, based on the classification of vasculitis proposed by Watts et al. [5]. After the cessation of PTU, the titers of both MPO-ANCA and PR3ANCA exhibited spontaneous reduction. Because the nasal septal perforation was destructive and had the potential to develop into a saddle nose, she desired treatment with high-dose prednisolone ( $1 \mathrm{mg} / \mathrm{kg} /$ day) and methotrexate (10 mg/week). She was treated with this regimen and discharged 1 month later. We are currently monitoring the nasal septal perforation by endoscopy and MRI, and progression has not yet been observed for 15 months. The titers of both MPO-ANCA and PR3-ANCA have been decreasing within our reference value (Figure 3 ).

\section{Discussion}

In this report, we present the case of a young woman who developed nasal septal perforation with a high titer of MPO-
ANCA and a low titer of PR3-ANCA following the use of PTU for 14 months. ANCA is regarded as a marker of small vessel vasculitis. However, some patients who test positive for ANCA exhibit infections (such as tuberculosis), inflammatory bowel diseases, other autoimmune disorders, and malignancies [6-10]. Moreover, positivity for both MPO-ANCA and PR3-ANCA is reported to appear in subacute bacterial endocarditis [11]. PTU is prescribed for treating GD and is a medicine that induces drug-induced AAV. It is reported that $15 \%-64 \%$ of patients taking PTU exhibit ANCA positivity and that one-third to one-fourth of patients taking PTU present some symptoms of AAV [12]. Drug-induced AAV usually manifests with mild symptoms, including rash, arthralgia, myalgia, fever, fatigue, or anorexia. However, in some cases, severe symptoms, such as alveolar hemorrhage or rapid progressive glomerulonephritis, may appear [12].

The manifestation in our patient was limited to nasal septal perforation. The abnormal findings of the paranasal sinus are reported to appear in $85 \%$ of GPA patients, $25 \%$ of whom present only these symptoms [13]. Nasal septal perforation is found in approximately $11 \%-33 \%$ of GPA patients and is identified by epistaxis in most of them [13-16]. The chronic use of vasoconstrictive nasal sprays or 


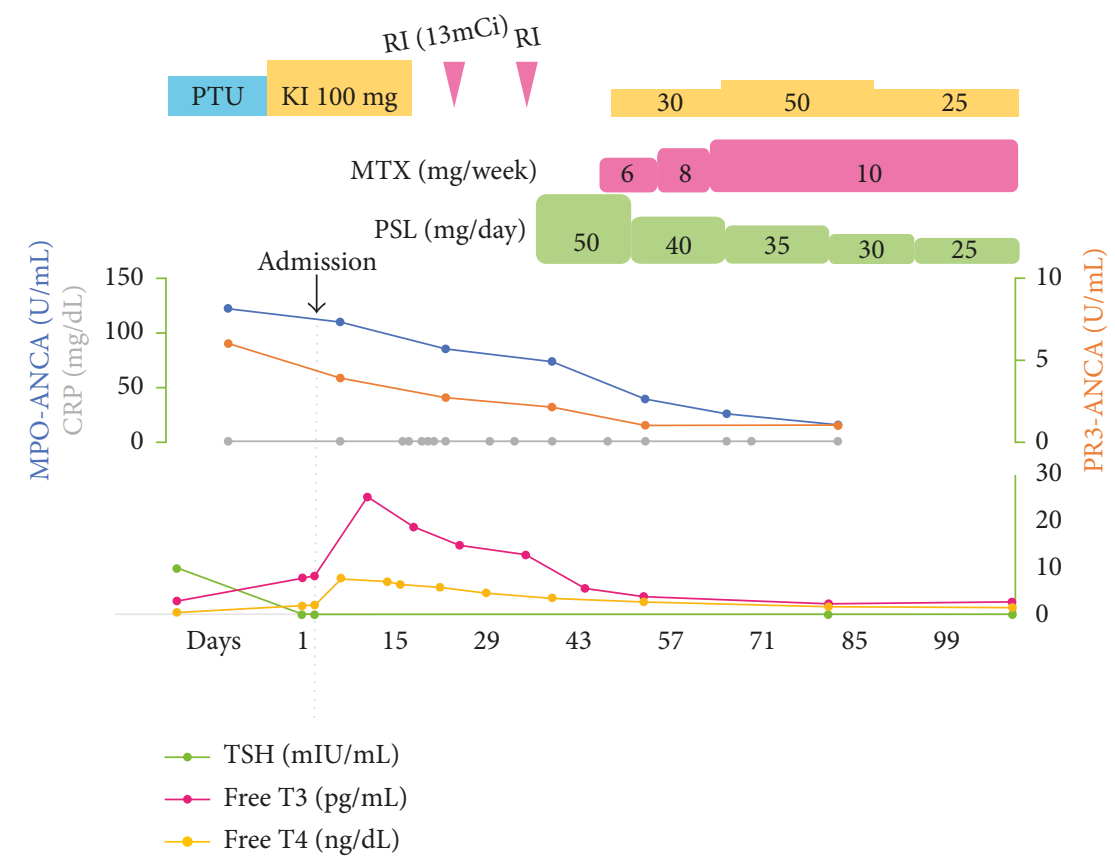

FIgURE 3: Clinical course. CRP: C-reactive protein; free T3: free triiodothyronine; free T4: free thyroxine; KI: potassium iodide; MPOANCA: myeloperoxidase-anti-neutrophil cytoplasmic antibody; MTX: methotrexate; PR3-ANCA: proteinase 3-anti-neutrophil cytoplasmic antibody; PSL: prednisolone; PTU: propylthiouracil; RI (13mCi): radioisotope (13mCi); TSH: thyroid-stimulating hormone.

cocaine may cause nasal septal perforation, and cocaine occasionally induces ANCA as well [17]. One case report presented a 26-year-old woman with PTU-induced AAV who manifested severe skin ulcer, pulmonary infiltrates, and nasal septal perforation [18]. To our knowledge, this is the only report presenting PTU-induced AAV with nasal septal perforation, which was observed in a patient with systemic involvement. Our case is different because her manifestation was limited to the nasal septal perforation. The prognosis of drug-induced AAV is better than that of primary AAV, and corticosteroid treatment can be steadily reduced and finally discontinued [19]. As demonstrated in this case, druginduced AAV is characterized by onset at a younger age, positivity for both MPO-ANCA and PR3-ANCA, and better prognosis than that of primary AAV [12].

In conclusion, GD patients who are taking PTU are at a risk of developing AAV. Nasal septal perforation is rare but can be the only manifestation of drug-induced AAV.

\section{Consent}

The authors obtained the informed consent from the patient.

\section{Conflicts of Interest}

The authors declare that they have no conflicts of interest.

\section{Acknowledgments}

The authors thank the staff of the Department of Hematology and Rheumatology, Tohoku University, for helpful discussions. This work was supported by JSPS KAKENHI
Grant no. 16H06642 for Tsuyoshi Shirai and a research grant from Kanae Foundation for Tsuyoshi Shirai.

\section{References}

[1] I. A. Mansi, A. Opran, and F. Rosner, "ANCA-associated small-vessel vasculitis," American Family Physician, vol. 65, no. 8, pp. 1615-1620, 2002.

[2] J. C. Jennette, R. J. Falk, P. A. Bacon et al., "2012 revised International Chapel Hill Consensus Conference Nomenclature of Vasculitides," Arthritis \& Rheumatism, vol. 65, no. 1, pp. 1-11, 2013.

[3] R. G. Grau, "Drug-induced vasculitis: new insights and a changing lineup of suspects," Current Rheumatology Reports, vol. 17, no. 12, p. 71, 2015.

[4] H. B. Burch and D. S. Cooper, "Management of Graves' disease a review," JAMA, vol. 314, no. 23, pp. 2544-2554, 2015.

[5] R. Watts, S. Lane, T. Hanslik et al., "Development and validation of a consensus methodology for the classification of the ANCA-associated vasculitides and polyarteritis nodosa for epidemiological studies," Annals of the Rheumatic Diseases, vol. 66, no. 2, pp. 222-227, 2007.

[6] I. Diamantopoulos II and N. S. Jones, "The investigation of nasal septal perforations and ulcers," Journal of Laryngology \& Otology, vol. 115, no. 7, pp. 541-544, 2001.

[7] T. Shirai, R. Takahashi, Y. Tajima et al., "Peripheral T cell lymphoma with a high titer of proteinase-3-antineutrophil cytoplasmic antibodies that resembled Wegener's granulomatosis," Internal Medicine, vol. 48, no. 23, pp. 2041-2045, 2009.

[8] L. F. Flores-Suárez, J. Cabiedes, A. R. Villa, F. J. van der Woude, and J. Alcocer-Varela, "Prevalence of antineutrophil cytoplasmic autoantibodies in patients with tuberculosis," Rheumatology, vol. 42, no. 2, pp. 223-229, 2003. 
[9] X. Bosch, A. Guilabert, and J. Font, “Antineutrophil cytoplasmic antibodies," The Lancet, vol. 368, no. 9533, pp. 404418, 2006.

[10] E. Houben, W. A. Bax, B. van Dam et al., "Diagnosing ANCAassociated vasculitis in ANCA positive patients: a retrospective analysis on the role of clinical symptoms and the ANCA titre," Medicine, vol. 95, no. 40, p. e5096, 2016.

[11] A. M. Tiliakos and N. A. Tiliakos, "Dual ANCA positivity in subacute bacterial endocarditis," Journal of Clinical Rheumatology, vol. 14, no. 1, pp. 38-40, 2008.

[12] M. Chen, Y. Gao, X. H. Guo, and M. H. Zhao, "Propylthiouracilinduced antineutrophil cytoplasmic antibody-associated vasculitis," Nature Reviews Nephrology, vol. 8, no. 8, pp. 476-483, 2012.

[13] J. Wojciechowska, W. Krajewski, P. Krajewski, and T. Kręcicki, "Granulomatosis with polyangiitis in otolaryngologist practice: a review of current knowledge," Clinical and Experimental Otorhinolaryngology, vol. 9, no. 1, pp. 8-13, 2016.

[14] E. Eren, T. Kalkan, S. Arslanoğlu et al., "Clinical factors associated with the diagnosis of granulomatosis with polyangiitis," Otolaryngology-Head and Neck Surgery, vol. 156, no. 3, pp. 484-488, 2017.

[15] S. P. McAdoo, A. Hall, J. Levy, A. D. Salama, and C. D. Pusey, "Proteinase-3 antineutrophil cytoplasm antibody positivity in patients without primary systemic vasculitis," Journal of Clinical Rheumatology, vol. 18, no. 7, pp. 336-340, 2012.

[16] S. B. Cannady, P. S. Batra, C. Koening et al., "Sinonasal Wegener granulomatosis: a single-institution experience with 120 cases," Laryngoscope, vol. 119, no. 4, pp. 757-761, 2009.

[17] M. Trimarchi, M. Bussi, R. A. Sinico, P. Meroni, and U. Specks, "Cocaine-induced midline destructive lesions-An autoimmune disease?," Autoimmunity Reviews, vol. 12, no. 4, pp. 496-500, 2013.

[18] B. D. Houston, M. E. Crouch, J. E. Brick, and A. G. DiBartolomeo, "Apparent vasculitis associated with propylthiouracil use," Arthritis \& Rheumatism, vol. 22, no. 8, pp. 925-928, 1979.

[19] S. Morita, Y. Ueda, and K. Eguchi, "Anti-thyroid druginduced ANCA-associated vasculitis: a case report and review of the literature," Endocrine Journal, vol. 47, no. 4, pp. $467-470,2000$ 


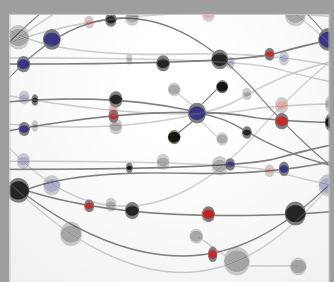

The Scientific World Journal
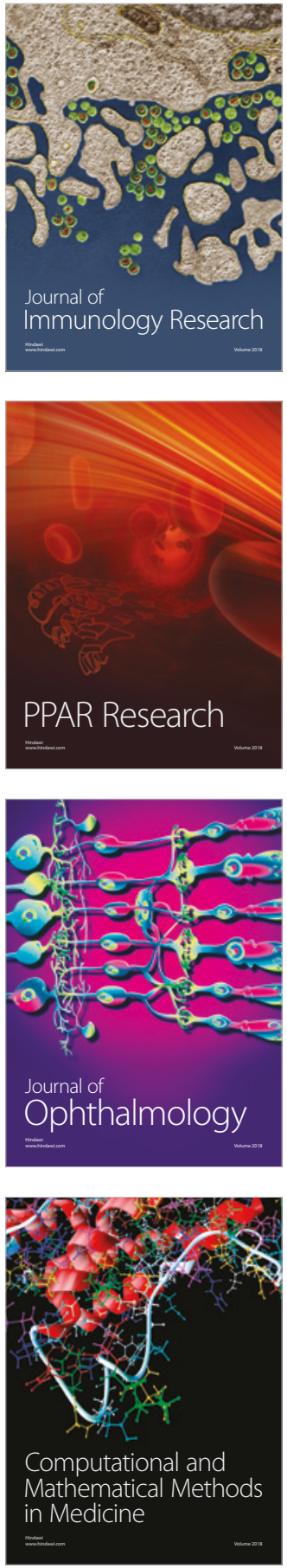

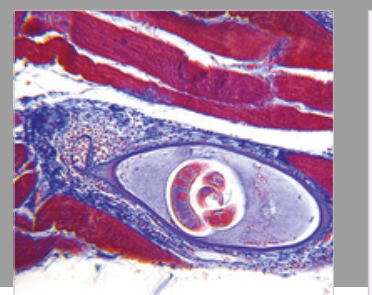

Gastroenterology Research and Practice

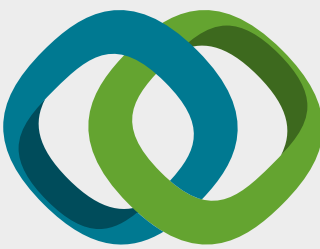

\section{Hindawi}

Submit your manuscripts at

www.hindawi.com
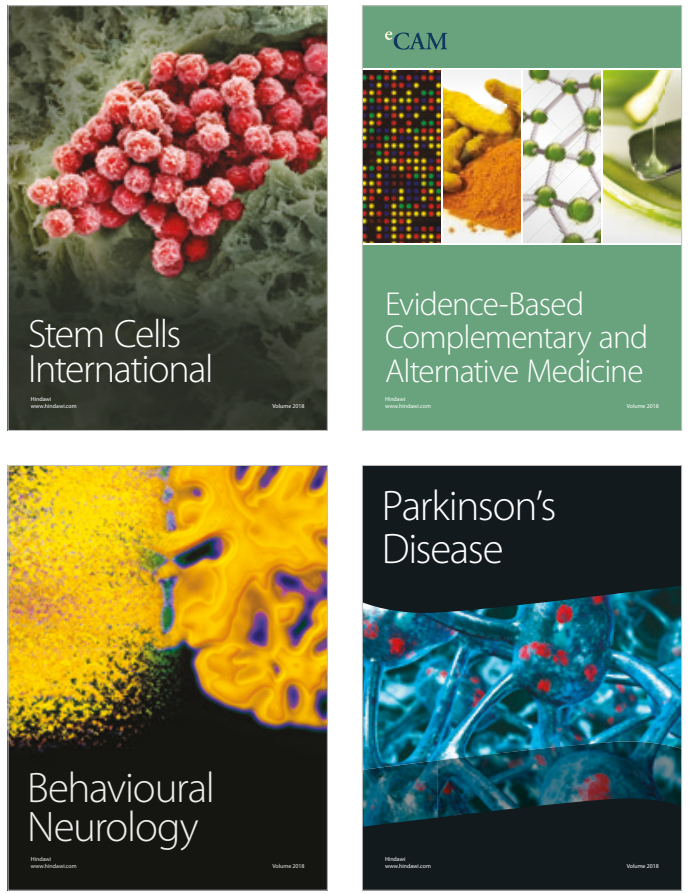

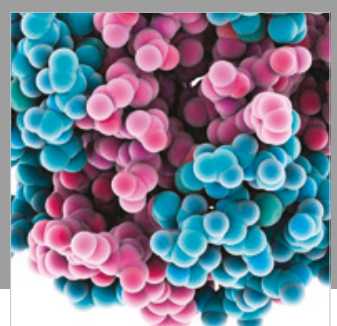

ournal of

Diabetes Research

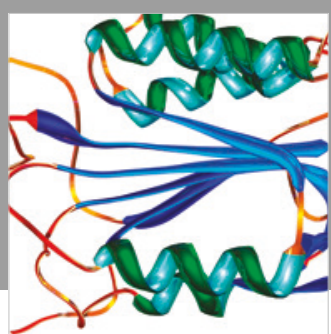

Disease Markers
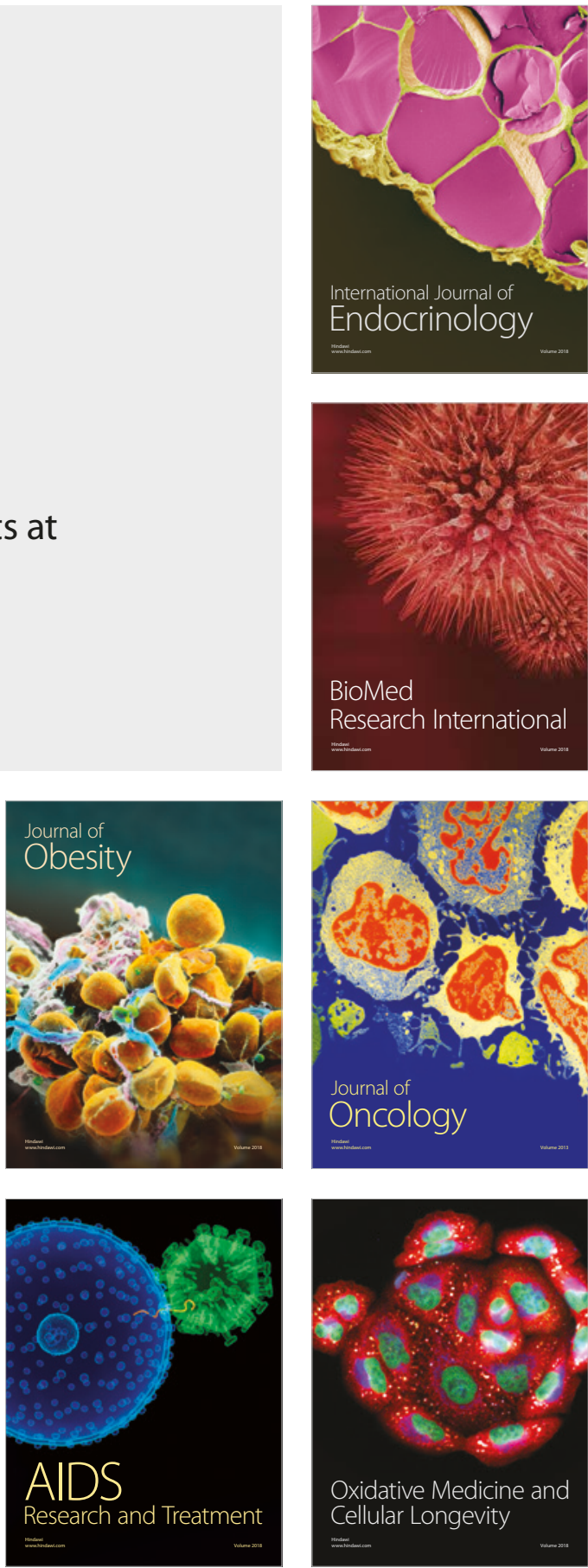